\title{
METOPROLOL-INDUCED LIVER INJURY AND THE HEPATOPROTECTIVE ROLE OF VITAMIN E IN RABBITS
}

\section{MAYSAA BANAY ZUBAIRI* \\ Department of Pharmacology and Toxicology, College of Pharmacy, University of Basra, Basra, Iraq. Email: phmaysaaalbasri@gmail.com} Received: 28 December 2018, Revised and Accepted: 25 January 2019

\section{ABSTRACT}

Objective: This study aims to investigate the hepatotoxicity of metoprolol and find a prophylactic way to protect the liver through the use of Vitamin E.

Methods: A total of 18 male rabbits were divided randomly into three groups, with six in each group. Group I (control) received corn oil, Group II was treated with metoprolol (20 mg/kg), and Group III was treated with Vitamin E (175 mg/kg) 30 min before metoprolol treatment. All treatments were given orally and daily for 14 days; animals were sacrificed at day 15 . Biochemical parameters were estimated, and the liver was used for histopathological examinations.

Results: Histopathological changes of liver injury were found in all metoprolol-treated rabbits, while these changes were minimized in all rabbits who were given Vitamin E. Serum liver function tests and malondialdehyde (MDA) were significantly elevated after metoprolol treatment and returned close to the control value on prior treatment with Vitamin E. Serum MDA, aspartate aminotransferase, and alanine aminotransferase were significantly decreased following treatment with Vitamin E.

Conclusion: Vitamin E may have a hepatoprotective effect against metoprolol-induced liver injury in rabbits.

Keywords: Metoprolol, Hepatic injury, Hydropic degeneration, Vitamin E, Antioxidants.

(C) 2019 The Authors. Published by Innovare Academic Sciences Pvt Ltd. This is an open access article under the CC BY license (http://creativecommons. org/licenses/by/4. 0/) DOI: http://dx.doi.org/10.22159/ajpcr.2019.v12i4.31813

\section{INTRODUCTION}

Drug-induced liver injury is a well-known adverse effect of numerous medications, with clinical presentation ranging from an elevation of asymptomatic liver enzymes through to liver failure [1]. A cardioselective beta1-adrenergic blocking agent, metoprolol, is used to treat a number of conditions including angina pectoris, acute myocardial infarction, heart failure, tachyarrhythmias, and mild-to-moderate hypertension, as well as being used as prophylaxis for migraine headaches $[2,3]$. Inactive metabolites are generated when metoprolol is metabolized as it undergoes $\alpha$-hydroxylation and 0-demethylation as an enzyme substrate of the cytochrome liver enzyme CYP2D6 and a small percentage by CYP3A4 [4]. CYP2D6 activity is notably variable among individuals due to the polymorphism of the CYP2D6 gene, so the plasma concentrations of metoprolol in poor metabolizers are several times higher than those found in extensive metabolizers [5].

Metoprolol has been linked to some clinical cases of drug-induced liver injury, with the first case report of metoprolol hepatotoxicity being reported in 1989. In this case, an elderly woman developed fatigue and abdominal pain 2 weeks after starting metoprolol (50 mg daily) for migraine headaches. Laboratory tests showed marked elevations in serum aminotransferase, while a liver biopsy revealed occasional ballooned hepatocytes without inflammation or fibrosis, together with moderate steatosis [6]. In 2017, Cyriac Philips reported the case of a young woman who was left with severe acute liver injury, jaundice, and Grade 1 hepatic encephalopathy following 8 weeks of metoprolol use to treat acute myocardial infarction. All the reported cases confirmed that liver injury gradually disappeared and liver test results improved when metoprolol was discontinued [7].

Antioxidant vitamins such as Vitamin C and Vitamin E play a key role in regulating a wide range of pathological and physiological processes. As well as enhancing the immune system and generating free radical scavenging properties, antioxidant vitamins are also involved with the alteration of cell proliferation and the modification of the carcinogen metabolism $[8,9]$. As a primary liposoluble antioxidant, Vitamin E is thought to play a pivotal role in scavenging free oxygen radicals and stabilizing cell membranes, which allows cell permeability to be maintained [10]. In addition, this critical vitamin has a defensive role in that it minimizes the harmful effects of oxidation that causes damage to the liver and other body parts [11]. Furthermore, Vitamin E has a notably positive effect on cell membranes as it has the effect of protecting the polyunsaturated fats within them, therefore, preserving the structure and function of the membranes [12].

In the present study, oxidative stress has been addressed as a mechanism that may be responsible for metoprolol-induced liver injury. However, no studies to date have studied the potentially protective nature of Vitamin E with regard to mitigating metoprolol-induced liver damage. Therefore, the author selected exogenous Vitamin E to evaluate its protective effects on metoprolol-induced liver injury in rabbits.

\section{MATERIALS AND METHODS}

\section{Chemicals and their preparation}

Metoprolol as metoprolol succinate tablet $(50 \mathrm{mg}$ ) was purchased from AstraZeneca, Switzerland. An accurate dose of $20 \mathrm{mg} / \mathrm{kg}$ was administered orally for each rabbit through a stainless steel feeding tube. Vitamin E as d-alpha tocopheryl acetate powder was purchased from Bulk Supplement.com, Henderson, USA. Using a standard measuring spoon, $175 \mathrm{mg}$ of Vitamin E was accurately measured and dissolved in $1 \mathrm{ml}$ of corn oil to prepare an accurate dose of $175 \mathrm{mg} / \mathrm{kg}$.

\section{Animals}

The experiment was carried out on 18 locally bred, 9-month-old domestic male rabbits weighing 1-2 kg. The animals were housed in the animal house at Basra College of Pharmacy; they were kept in stainless steel cages for acclimatization, with free access to food and water and a 12:12-h cycle of light and darkness. The animals were not fed for 
12 hours before the experiment. The study was approved by the Local Institutional Ethical Committee of the Faculty of Pharmacy College at the University of Basra.

\section{Study protocol}

The study was carried out between January and April 2018. The rabbits were randomly divided into three groups, with six animals in each group, as follows:

- Group I: Served as the control group, received $1 \mathrm{ml}$ of corn oil daily for 14 days.

- Group II: Treated with a single oral dose of metoprolol (20 mg/kg/d) for 14 days.

- Group III: Pre-treated with Vitamin E (175 mg/kg/d) orally, 30 min before metoprolol treatment.

On the $15^{\text {th }}$ day of drug administration, the animals were anesthetized under light ether anesthesia. Blood was taken directly from the heart and transferred into a non-heparinized tube; then, the serum was separated through centrifugal spinning for $20 \mathrm{~min}$ at $3000 \mathrm{rpm} .1 \mathrm{ml}$ of serum was taken for serum malondialdehyde (MDA) measurement and the remainder was frozen at $-20^{\circ} \mathrm{C}$ for the analysis of liver function test (LFT) measurements. The rabbits were then sacrificed, and liver specimens were taken for histopathological examination.

\section{Laboratory measurements}

Determination of the serum MDA level: Thiobarbituric acid assay of Buege and Aust (1978) was used for measuring serum MDA [13].

LFT: The activities of aspartate aminotransferase (AST), alanine aminotransferase (ALT), alkaline phosphatase (ALP), and total bilirubin in serum were estimated by an autoanalyzer (Biochemistry Analyzer, Integra 400 Plus, Roche, Germany), by selecting the already coded program in the analyzer for all samples.

\section{Histopathological examination}

The livers were carefully removed and washed with $0.9 \%$ saline solution. The liver specimens were kept in $10 \%$ formalin and embedded in paraffin wax before being cut into $5 \mu \mathrm{m}$ slices. The slices were stained with hematoxylin and eosin ( $\mathrm{H}$ and $\mathrm{E})$; then, the specimens were examined by a specialist histopathologist at the Department of Pathology and Forensic Medicine, College of Medicine, Basra. The examiner was blind for the treatments.

\section{Statistical analysis}

The results were expressed as mean \pm standard deviation. The data were statistically evaluated using ordinary one-way analysis of variance followed by Tukey's multiple comparisons test using GraphPad Prism 8 computer software. The results were considered statistically significant at $\mathrm{p}<0.05$

\section{RESULTS}

Effect of treatments on serum liver enzymes

In Table 1, it is shown that metoprolol treatment produced significant elevation $(\mathrm{p}<0.01)$ in the levels of ALT, AST, and ALP, compared to the control group. Pre-treatment with Vitamin E significantly $(p<0.01)$ decreased ALT and AST levels, while the reduction in the ALP level did not achieve statistical significance compared with the value of metoprolol treatment $(\mathrm{p}=0.6)$.

\section{Effect of treatments on serum total bilirubin}

The serum total bilirubin in the control group was significantly increased $(\mathrm{p}<0.01)$ by metoprolol treatment, then decreased by Vitamin $\mathrm{E}$; this reduction was statistically insignificant $(\mathrm{p}=0.17)$ as presented in Table 1.

\section{Effect of treatments on serum MDA}

The serum level of MDA significantly increased $(p<0.0001)$ in the group treated with metoprolol compared with the control group. As illustrated in Table 1, treatment with Vitamin E before metoprolol significantly reduced the MDA level $(\mathrm{p}<0.0001)$

\section{Histopathological examination}

\section{Control group}

None of the liver samples obtained from rabbits in this group showed any histopathological changes $(\mathrm{n}=6)$. A representative histopathological slide is presented in Fig. 1a.

\section{Metoprolol group}

All of the rabbits in the experiment demonstrated significant liver injury after the metoprolol challenge; in all rabbits, the liver showed severe hydropic degeneration, perivenular sinusoidal dilatation, and moderate-to-severe inflammation represented by lymphocytic infiltration (portal and lobular) (Fig. 1b and c).

\section{Metoprolol+Vitamin E group}

Histopathological examination showed amelioration of metoprololinduced liver injury in all the rabbits that had been pre-treated with Vitamin E. All these rabbits' livers showed hydropic degeneration that ranged from mild to moderate. Mild perivenular sinusoidal dilatation was seen in three rabbits, while two rabbits were found to have mild inflammatory cell infiltration of lymphocytes in the portal area (Fig. 1d and inset).

\section{DISCUSSION}

As a beta-blocker, metoprolol is used in the treatment of angina, heart failure, and hypertension [14]. While metoprolol therapy has been associated with a low rate of mild-to-moderate elevation of the level of serum liver enzymes, these are usually asymptomatic. When liver injury occurs due to metoprolol therapy, this varies in severity and ranges from mild serum aminotransferase elevations to mild acute hepatitis with jaundice [6].

The mechanism behind metoprolol-induced liver injury is unknown, but in the present study, the oxidative stress mechanism is implicated in the pathogenesis of certain injury induced by metoprolol. This is because the susceptibility of an organ to oxidative stress is a role of the overall balance between the factors that induce oxidative stress and those that exhibit antioxidant capability [15]

MDA concentrations always increase with elevated lipid peroxidation [16], and this experiment determined to investigate whether metoprolol caused oxidative damage. The significant elevation of MDA following the introduction of metoprolol indicates that it is possible that metoprolol interacts with vital subcellular sites including the mitochondria cytosol and peroxisomes, which results

Table 1: Effect of Vitamin E on serum hepatic marker enzymes, total bilirubin, and MDA in metoprolol-induced liver injury in rabbits

\begin{tabular}{lllll}
\hline Groups & ALT $(\mathbf{U} / \mathbf{L})$ & AST $(\mathbf{U} / \mathbf{L})$ & ALP $(\mathbf{U} / \mathbf{L})$ & TB $(\mathbf{m g} / \mathbf{d l})$ \\
\hline Control & $32.01 \pm 4.70$ & $22.05 \pm 4.04$ & $89.06 \pm 3.57$ & $0.883 \pm 0.25$ \\
Metoprolol & $45.68 \pm 5.22$ & $41.68 \pm 5.53$ & $111.8 \pm 12.21$ & $0.341 \pm 0.05$ \\
Metoprolol + Vitamin E & $33.63 \pm 5.21^{* *}$ & $25 \pm 5.72^{* *}$ & $106.8 \pm 11.78$ & $1.616 \pm 0.41$ \\
\hline
\end{tabular}

$\mathrm{n}=6$, Data expressed as mean \pm SD. P value significantly different from the metoprolol value, ${ }^{* *} \mathrm{p}<0.01,{ }^{* * *} \mathrm{p}<0.0001$. ALT: Alanine aminotransferase, AST: Aspartate aminotransferase, ALP: Alkaline phosphatase, TB: Total bilirubin, MDA: Malondialdehyde, SD: Standard deviation 


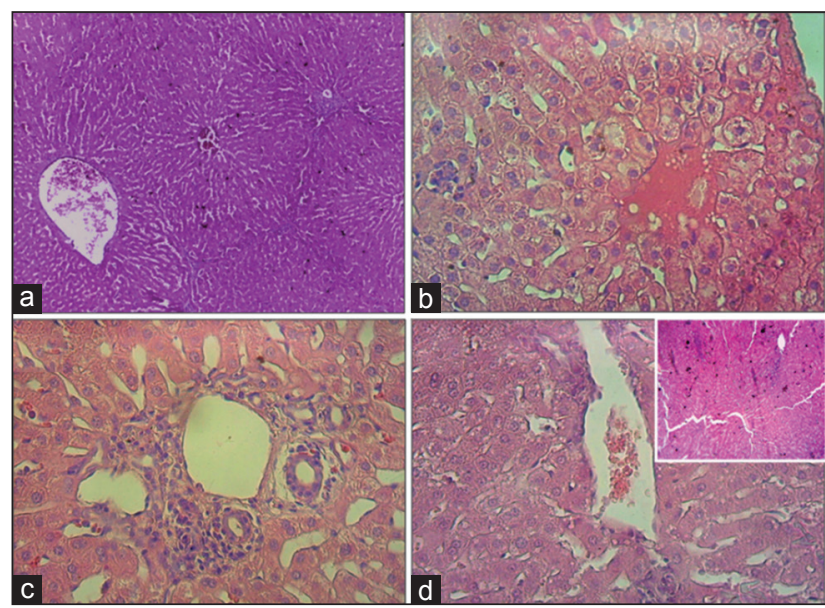

Fig. 1: Liver histopathology (H and E stain). (a) Healthy liver tissue from a rabbit in the control group (corn oil); a clear area around the central vein is observed (power $\times 40$ ). ( $b$ and c) Metoprolol-treated group $20 \mathrm{mg} / \mathrm{kg}$ (power $\times 10$ ) congested central vein surrounded by hepatocytes with severe hydropic degeneration, sinusoidal dilatation, and periportal tract inflammation. (d and inset) Vitamin E $175 \mathrm{mg} / \mathrm{kg}$ followed by metoprolol $20 \mathrm{mg} / \mathrm{kg}$ (power $\times 10$ and 40 , respectively)

in the generation of extreme amounts of free radicals. These free radicals generate oxidative damage to biomolecules such as lipids and proteins, which causes oxidative stress and may contribute to various pathophysiological changes including hydropic degeneration of hepatocytes. Lipid peroxidation is one of the main manifestations of metoprolol-induced oxidative damage. It is possible that the significant increase in the level of serum MDA in metoprolol-treated rabbits could be due to excessive formation of free radicals, which leads to the deterioration of biological macromolecules [17]. Vitamin E $(175 \mathrm{mg} / \mathrm{kg})$ recovers the body's antioxidant capacity and protects the liver by decreasing lipid peroxidation, represented by the significant reduction in serum MDA. These findings provide a theoretical basis for the prevention of toxicity caused by metoprolol, which may benefit humans.

The alterations in the hepatocytes after exposure to metoprolol in this study were mainly hydropic change and cellular edema. Hydropic change is an acute reversible change resulting from non-lethal injuries; it is an intracytoplasmic accumulation of water due to the incapacity of the cells to maintain the fluid and ionic homeostasis [18]. This can be clearly observed in the livers shown in Fig. 1b and c. Metoprolol may induce hydropic change indirectly through the release of extremely reactive and toxic free radicals. These free radicals cause lipid peroxidation, as well as cell membrane damage, with the cellular swelling resulting from an inundation of sodium and water. Metoprolol treatment in the rabbits appeared to cause the vacuolated swelling of the cytoplasm in the hepatocytes, which may be indicative of an acute liver injury.

Several previous experimental research studies on animal species have demonstrated that Vitamin E treatment ameliorated liver injury that had been induced by exposure to carbon tetrachloride [19], doxorubicin, anthracycline antibiotic [20], azathioprine [21], and pesticides such as malathion [22]. In addition, many studies have proved that Vitamin E supplements can decrease steatosis, inflammation, and cell injury in patients with non-alcoholic fatty liver disease. Furthermore, the antioxidant effect of Vitamin E may reduce liver damage caused by oxidation [23].

Oral pre-treatment with Vitamin E $(175 \mathrm{mg} / \mathrm{kg}) 30 \mathrm{~min}$ before daily metoprolol administration was reported to ameliorate histopathological changes. Investigators demonstrated that the mechanisms underlying the amelioration of liver injury by Vitamin E appeared to be related not only to the prevention of liver cell deterioration but also to the maintenance of membrane integrity. The molecules of the Vitamin E family exert anti-inflammatory activities [23]. Therefore, liver inflammation either changed to mild following Vitamin E treatment or disappeared entirely.

The elevation in the serum levels of hepatic marker enzymes and total bilirubin was attributed to damaged liver cells since hepatic enzymes are located in the cytosol and released into the blood following liver damage $[24,25]$. After Vitamin E administration, there was a reduction in lipid peroxidation and the integrity of hepatocytes was maintained, while there was less leakage of liver enzymes from hepatocytes. This led to decreased serum enzymes levels, particularly ALT and AST. The nonsignificant reduction in total bilirubin agreed with many other studies that have described the beneficial effect of Vitamin E on liver function. It is likely that this is related to a reduction in red cell hemolysis and enhancement of bilirubin conjugation [26-29].

\section{CONCLUSION}

The results of the current study revealed that metoprolol $(20 \mathrm{mg} / \mathrm{kg} / \mathrm{d}$ for 14 days) caused lipid peroxidation, significant elevation in the serum liver enzymes, and histopathological changes in the liver. Supplementation with Vitamin E exhibited a moderate protective effect. The appearance of hepatocytes after metoprolol treatment suggests that metoprolol interacts indirectly with the hepatic tissue by interfering with the antioxidant defense mechanism, leading to reactive oxygen species generation. In turn, this may induce stress in the hepatocytes, which undergo severe hydropic degeneration, perivenular sinusoidal dilatation, and moderate-to-severe inflammation.

Vitamin E (175 mg/kg half an hour before metoprolol) significantly reduced the serum levels of ALT, AST, and MDA. In addition, Vitamin E showed mild-to-moderate hydropic degeneration of all rabbits' livers and mild inflammation in two rabbits only, while it improved the hepatic histopathological damage induced by metoprolol. Moreover, the free radical scavenging activity of Vitamin E decreased lipid peroxidation and thus oxidative stress, and it, therefore, exerted protection against metoprolol-induced liver injury.

\section{AUTHORS' CONTRIBUTIONS}

Maysaa Banay Zubairi designed and performed the experiment, analyzed the data, and wrote the manuscript.

\section{CONFLICTS OF INTEREST}

The author declares that there are no conflicts of interest regarding publication of this article.

\section{REFERENCES}

1. Hansen T, Fynne L. Jaundice and liver injury with cholestatic pattern after treatment with metoprololsuccinat. Ugeskr Laeger 2017; 179:V09170698.

2. Walter E, McKinlay J, Corbett J, Kirk-Bayley J. Review of management in cardiotoxic overdose and efficacy of delayed intralipid use. J Intensive Care Soc 2018;19:50-5.

3. Peters DH, Benfield P. Metoprolol: A pharmacoeconomic and qualityof-life evaluation of its use in hypertension, post-myocardial infarction and dilated cardiomyopathy. Pharmacoeconomics 1994;6:370-400.

4. Blake CM, Kharasch ED, Schwab M, Nagele P. A meta-analysis of CYP2D6 metabolizer phenotype and metoprolol pharmacokinetics. Clin Pharmacol Ther 2013;94:394-9.

5. Rau T, Heide R, Bergmann K, Wuttke H, Werner U, Feifel N, et al. Effect of the CYP2D6 genotype on metoprolol metabolism persists during long-term treatment. Pharmacogenetics 2002;12:465-72.

6. Larrey D, Henrion J, Heller F, Babany G, Degott C, Pessayre D, et al. Metoprolol-induced hepatitis: Rechallenge and drug oxidation phenotyping. Ann Intern Med 1988;108:67-8.

7. Philips C, Paramaguru R, Mahadevan P, Ravindranath J, Augustine P. Metoprolol-induced severe liver injury and successful management with therapeutic plasma exchange. Cureus 2017;9:e1209.

8. Khaidakov M, Bishop ME, Manjanatha MG, Lyn-Cook LE, Desai VG, 
Chen JJ, et al. Influence of dietary antioxidants on the mutagenicity of 7,12-dimethylbenz[a] anthracene and bleomycin in female rats. Mutat Res 2001;480-481:163-70.

9. Dahdouh F, Attalah S, Djabar MR, Kachrid Z. Effect of the joint supplementation of Vitamin $\mathrm{C}$ and Vitamin $\mathrm{E}$ on nickel heamatotoxicity and nephrotoxicity in male Swiss albino mice. Int J Pharm Pharm Sci 2016;8:234-9.

10. Ognjanović BI, Pavlović SZ, Maletić SD, Zikić RV, Stajn AS, Radojicić RM, et al. Protective influence of Vitamin E on antioxidant defense system in the blood of rats treated with cadmium. Physiol Res 2003;52:563-70.

11. Schmölz L, Birringer M, Lorkowski S, Wallert M. Complexity of Vitamin E metabolism. World J Biol Chem 2016;7:14-43.

12. Ibrahim MA, AL-Shawi NN. Effects of Vitamin E and coenzyme Q10 supplementation against doxorubicin-induced neurotoxicity in rats. Asian J Pharm Clin Res 2018;11:402-6.

13. Buege JA, Aust SD. Microsomal lipid peroxidation. Methods Enzymol 1978;52:302-10

14. Niharika V. Introduction to hyperlipidemia and its treatment: A review. Int J Curr Pharm Res 2017;9:6-14.

15. Chow CK. Nutritional influence on cellular antioxidant defense systems. Am J Clin Nutr 1979;32:1066-81.

16. Drake IM, Mapstone NP, Schorah CJ, White KL, Chalmers DM, Dixon MF, et al. Reactive oxygen species activity and lipid peroxidation in Helicobacter pylori associated gastritis: Relation to gastric mucosal ascorbic acid concentrations and effect of $H$ pylori eradication. Gut 1998;42:768-71.

17. Palati DJ, Vanapatla SR. Protective role of Aerva monsoniae and selenium on cadmium-induced oxidative liver damage in rats. Asian J Pharm Clin Res 2018;11:177-81.

18. Almansour MI, Jarrar YB, Jarrar BM. In vivo investigation on the chronic hepatotoxicity induced by sertraline. Environ Toxicol Pharmacol 2018;61:107-15.

19. Khalaf AA, Mekawy ME, Moawad MS, Ahmed AM. Comparative study on the protective effect of some antioxidants against $\mathrm{Ccl} 4$ hepatotoxicity in rats. Egypt J Nat Toxins 2009;6:59-82

20. Gokcimen A, Cim A, Tola HT, Bayram D, Kocak A, Ozgüner F, et al. Protective effect of $\mathrm{N}$-acetylcysteine, caffeic acid and Vitamin $\mathrm{E}$ on doxorubicin hepatotoxicity. Hum Exp Toxicol 2007;26:519-25.

21. Amouoghli-Tabrizi B, Mohajeri D, Mousavi G, Farajzade F, Khodadadi A, Alizade SB, et al. Biochemical and pathological study of protective effect of Vitamin $\mathrm{E}$ in azathioprine induced hepatotoxicity in rat. J Biol Sci 2009;9:339-44.

22. Kalender S, Ogutcu A, Uzunhisarcikli M, Accikgoz F, Durak D, Ulusoy $\mathrm{Y}$, et al. Diazinon-induced hepatotoxicity and protective effect of Vitamin $\mathrm{E}$ on some biochemical indices and ultrastructural changes. Toxicology 2005;211:197-206.

23. El Hadi H, Vettor R, Rossato M. Vitamin E as a treatment for nonalcoholic fatty liver disease: Reality or myth? Antioxidants (Basel) 2018;7:E12.

24. Cigremis Y, Turel H, Adiguzel K, Akgoz M, Kart A, Karaman M, et al. The effects of acute acetaminophen toxicity on hepatic mRNA expression of SOD, CAT, GSH-px, and levels of peroxynitrite, nitric oxide, reduced glutathione, and malondialdehyde in rabbit. Mol Cell Biochem 2009;323:31-8.

25. Zubairi MB, Ahmed JH, Al-Haroon SS. Effect of adrenergic blockers, carvedilol, prazosin, metoprolol and combination of prazosin and metoprolol on paracetamol-induced hepatotoxicity in rabbits. Indian $\mathrm{J}$ Pharmacol 2014;46:644-8.

26. Gross SJ. Vitamin $\mathrm{E}$ and neonatal bilirubinemia. Pediatrics 1979;64:321-3.

27. Jamshidi HR, Ghaneie M, Tabibian N. The effect of antioxidant (Vitamin E) administration on red blood cell fragility in $\beta$-thalassemia major. Iran J Blood Transfus 1997;4:134-8.

28. Sethi S, Prakash O, Pant A, Batra M, Kumar M. Hepatoprotective and antioxidant activity of Alpinia malaccensis roscoe rhizome. Int J Pharm Pharm Sci 2014;7:220-4

29. Kadeche L, Bourogaa E, Saoudi M, Boumendjel A, Djeffal A, Feki A, et al. Ameliorative effects of vanillin against metribuzin-induced oxidative stress and toxicity in rats. Int J Pharm Pharm Sci 2016;9:56-2. 